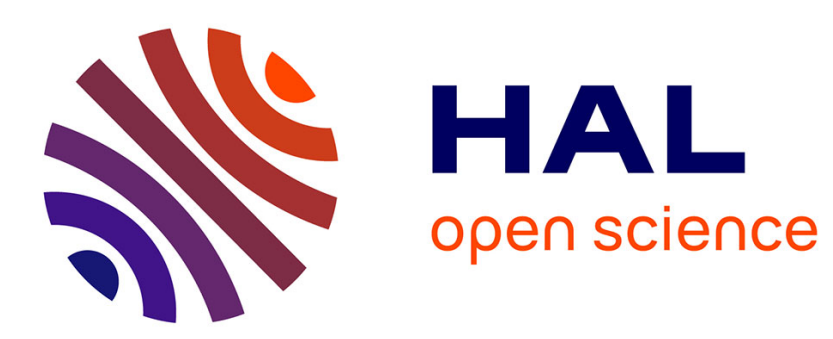

\title{
Variational convergences of dual energy functionals for elastic materials with a $\epsilon$ thin strong inclusion
}

Anne-Laure Bessoud, Giuseppe Geymonat, Francoise Krasucki, Gérard

Michaille

\section{- To cite this version:}

Anne-Laure Bessoud, Giuseppe Geymonat, Francoise Krasucki, Gérard Michaille. Variational convergences of dual energy functionals for elastic materials with a $\epsilon$ thin strong inclusion. Journal of Elasticity, 2012, 109, pp.51-65. 10.1007/s10659-011-9368-8 . hal-00790717

\section{HAL Id: hal-00790717 https://hal.science/hal-00790717}

Submitted on 16 Mar 2014

HAL is a multi-disciplinary open access archive for the deposit and dissemination of scientific research documents, whether they are published or not. The documents may come from teaching and research institutions in France or abroad, or from public or private research centers.
L'archive ouverte pluridisciplinaire HAL, est destinée au dépôt et à la diffusion de documents scientifiques de niveau recherche, publiés ou non, émanant des établissements d'enseignement et de recherche français ou étrangers, des laboratoires publics ou privés. 


\title{
Variational convergences of dual energy functionals for elastic materials with a $\varepsilon$-thin strong inclusion
}

\author{
Anne-Laure Bessoud · Giuseppe Geymonat · \\ Françoise Krasucki • Gérard Michaille
}

the date of receipt and acceptance should be inserted later

\begin{abstract}
We give a new derivation, based on the complementary energy formulation, of a simplified model for a multi-structure made up of two anisotropic hyper-elastic bodies connected by a thin strong material layer. The model is obtained by identifying the Mosco-limit of the stored complementary energy functional when the thickness is of order $\varepsilon$ and the stiffness of order $1 / \varepsilon$ where $\varepsilon$ is a positive real adimensional parameter. In order to prove the existence of the displacement associated with the stress we use a suitable weak version of the Saint-Venant compatibility condition also known as Donati's theorem.
\end{abstract}

AMS subject classifications: 49J45, 49N15, 74K30

Keywords: complementary elastic energy, asymptotic analysis, dual method, $\Gamma$-convergence, multistructure.

\footnotetext{
Anne-Laure Bessoud

Institut de Mathématiques de Toulon, EA-CNRS 2134, Université de Toulon et du Var, Avenue de l'Université, BP 132, 83957 La Garde Cedex, France

E-mail: annelaure.bessoud@gmail.com

Giuseppe Geymonat

Laboratoire de Mécanique des Solides, UMR-CNRS 7649

École Polytechnique

91128 PALAISEAU Cedex, France

E-mail: giuseppe.geymonat@lms.polytechnique.fr

Françoise Krasucki

I3M, UMR-CNRS 5149 , Université Montpellier II, Case courier 051,

Place Eugène Bataillon, 34095 Montpellier Cedex 5, France

E-mail: krasucki@math.univ-montp2.fr

Gérard Michaille

I3M , UMR-CNRS 5149, Université Montpellier II and MIPA Université de Nîmes, Case courier 051,

Place Eugène Bataillon, 34095 Montpellier Cedex 5, France

E-mail: micha@math.univ-montp2.fr
} 


\section{Introduction}

The modeling of complex structures obtained by assembling simpler elements with very different geometric and/or material characteristics is attracting the interest of engineers and applied mathematicians since it is a source of a variety of problems of practical importance. Many papers have been published in the last two-three decades to apply rigorous asymptotic methods in order to obtain a rational simplification of the modeling of complex structures obtained by joining elements of different dimensions and/or materials with highly contrasted properties. The first modeling of junctions between elements of different dimensions is due to $[10,19]$. The influence of a thin layer of highly contrasted rigidity inserted between two elastic material has been studied in various situations, see for instance $[23,6,7,24,16]$. In every situation the authors obtain a limit problem whose solution can be "easily" computed since a "material surface" substitutes the thin layer. This material surface carries a suitable surface energy density obtained through the limiting process from the global volume energy of the thin layer. Approaches based on the $\Gamma$-convergence of energy functionals (introduced by De Giorgi in 1975 , [11], for a very general class of functionals) have also been used to derive, from the original three dimensional problems, a variational limit problem taking into account the magnitude orders of both thickness and material coefficients, see for instance $[1,14,20,16,5]$.

Let us recall that the displacement-traction problem of three-dimensional linearized hyper-elasticity can be formulated as two different minimization problems, depending on whether the displacement vector field, or the stress tensor field is the unknown. If the unknown is the displacement vector field the minimization problem constitutes the modern version of the classical principle of minimum potential energy (for a historical perspective, see Gurtin [18] or Benvenuto [4]) also known as the primal formulation. If the unknown is the stress tensor field the minimization problem constitutes the modern version of the classical principle of minimum complementary energy (for a historical perspective, see again Gurtin [18] or Benvenuto [4]) also known as the dual formulation. Under some well-known positivity and regularity assumption on the elasticity tensor $\boldsymbol{C}$ or on the corresponding compliance elasticity tensor $\boldsymbol{A}$ each problem has a unique solution; moreover it is usually proved, see e. g. [12], that the stress $\boldsymbol{\sigma}=\boldsymbol{C u}$ associated with the primal solution $\boldsymbol{u}$ is also a solution (and hence the solution) of the dual problem. More difficult is to prove directly, as it is done in section 2, that there exists a displacement vector field $\boldsymbol{u}$ associated with $\boldsymbol{A} \boldsymbol{\sigma}$ where $\boldsymbol{\sigma}$ is the solution of the dual problem. Then this displacement vector field is also a solution (and hence the solution) of the primal problem. To this aim one verifies that the symmetric tensor field $\boldsymbol{A} \boldsymbol{\sigma}$ satisfies a weak form of the Saint Venant compatibility equations, also known as the Donati theorem ( see [18] sect. 18, [15] and, for a brief history of this result, section 7 in [8]).

All the previously quoted papers on the modeling of complex structures use the displacement vector field as the unknown for both the original and the limit problems and $\Gamma$-convergence is applied to the potential energy. The aim of this paper is to apply, in the framework of linear elasticity, a type of $\Gamma$-convergence (more precisely the so-called Mosco-convergence introduced by Mosco in 1969, [21], for convex functionals), to the complementary energy of a three layers elastic system where a thin and strong material is inserted between two elastic materials and to compare this limit problem with the limit problem obtained for the same situation with the primal formulation in [5]. Let us remark that in [3] an analogous situation is considered for a scalar case. However the method is different since it uses in an essential way a mixed formulation through a suitable lagrangian and a priori uses the convergence of the solutions of the primal problem. In a future paper we will use our direct approach to non-linear problems where only the complementary energy is given explicitly, e.g. for Norton-Hoff type materials.

After the presentation of the problem we apply in section 3 the usual rescaling procedure to the complementary energy formulation and in the following section we prove the Mosco convergence of the family of rescaled complementary energies to a suitable limit energy. At last in section 5 using an ad hoc Donati's theorem we prove the existence of a displacement vector field $\boldsymbol{u}$ associated with the minimum $\boldsymbol{\sigma}$ of the Mosco-limit functional, and that $\boldsymbol{u}$ is the solution of the $\Gamma$-limit problem obtained in $[5]$. 
The conventions of sum over repeated indexes and of comma representing derivative will be employed. Furthermore, $\mathbb{M}_{\text {sym }}^{3}$ denotes the space of symmetric $3 \times 3$ matrices, Latin indexes range in $\{1,2,3\}$ and Greek indexes range in $\{1,2\}$.

\section{A preliminary classical result in linear elasticity}

Let us consider a three-dimensional Euclidian space identified with $\mathbb{R}^{3}$ and such that the three vectors $\mathbf{e}_{i}$ form an orthonormal basis. Let $\Omega$ be a non-empty open subset of $\mathbb{R}^{3}$ with Lipschitz continuous boundary $\Gamma$, whose outer normal is denoted by $\mathbf{n}$. We assume that $\Gamma_{F}$ and $\Gamma_{0}$ are disjoint, relatively open subsets of $\Gamma$, that $\Gamma=\overline{\Gamma_{F}} \bigcup \overline{\Gamma_{0}}$ and $\mathcal{H}^{2}\left(\Gamma_{0}\right)>0$.

We look for the equilibrium of a linearly hyperelastic body occupying the open set $\Omega$, submitted to a system of volume force $\boldsymbol{f}$, to a surface density of forces $\boldsymbol{F}$ on $\Gamma_{F}$ and to a fixed displacement $\boldsymbol{u}_{0}$ on $\Gamma_{0}$. The solution of such equilibrium problem can be classically, see e. g. [18], characterized as the stress field $\boldsymbol{\sigma}$ realizing the minimum of the complementary energy :

$$
\Psi(\boldsymbol{\tau}):=\frac{1}{2} \int_{\Omega} \boldsymbol{A} \boldsymbol{\tau}: \boldsymbol{\tau} d x-\int_{\Gamma_{0}} \boldsymbol{\tau} \cdot \mathbf{n} \cdot \boldsymbol{u}_{0} d \mathcal{H}^{2}(\Gamma)
$$

on the space of the statically admissible stresses $\Sigma_{a d}:=\left\{\boldsymbol{\tau} ; \operatorname{Div} \boldsymbol{\tau}+\boldsymbol{f}=\mathbf{0}\right.$ in $\Omega, \boldsymbol{\tau} \mathbf{n}=\boldsymbol{F}$ on $\left.\Gamma_{F}\right\}$, where $\boldsymbol{A}$ is the compliance fourth-order tensor, satisfying the usual symmetry, regularity, boundedness and positivity assumptions.

In order to recast this result in a functional framework let us recall that in the well-known approach of Lions-Magenes it has been proved, see e. g. [17], that when $\boldsymbol{\tau} \in H(\operatorname{Div} ; \Omega):=\{\boldsymbol{\tau} \in$ $L^{2}\left(\Omega ; \mathbb{M}_{\text {sym }}^{3}\right) ;$ Div $\left.\boldsymbol{\tau} \in L^{2}\left(\Omega ; \mathbb{R}^{3}\right)\right\}$ then $\boldsymbol{\tau} \mathbf{n} \in H^{-\frac{1}{2}}\left(\Gamma ; \mathbb{R}^{3}\right)$ and moreover the following Green's formula holds for all $\boldsymbol{u} \in H^{1}\left(\Omega ; \mathbb{R}^{3}\right)$ and $\boldsymbol{\tau} \in H(\operatorname{Div} ; \Omega)$ :

$$
\int_{\Omega} \boldsymbol{\tau}: \boldsymbol{e}(\boldsymbol{u}) d x+\int_{\Omega} \operatorname{Div} \boldsymbol{\tau} \boldsymbol{u} d x=\langle\boldsymbol{\tau} \mathbf{n}, \boldsymbol{u}\rangle_{\Gamma}
$$

where $\langle., .\rangle_{\Gamma}$ denotes the duality between $H^{-\frac{1}{2}}\left(\Gamma ; \mathbb{R}^{3}\right)$ and $H^{\frac{1}{2}}\left(\Gamma ; \mathbb{R}^{3}\right)$ and, as usual, $\boldsymbol{e}(\boldsymbol{u})=\left(e_{i j}(\boldsymbol{u})\right)$ with $e_{i j}(\boldsymbol{u}):=\frac{1}{2}\left(\partial_{i} u_{j}+\partial_{j} u_{i}\right)$.

Let us assume that $\boldsymbol{f} \in L^{2}\left(\Omega ; \mathbb{R}^{3}\right)$ and that there exists $\widetilde{\boldsymbol{F}} \in H^{-\frac{1}{2}}\left(\Gamma ; \mathbb{R}^{3}\right)$ such that $\left.\widetilde{\boldsymbol{F}}\right|_{\Gamma_{F}}=\boldsymbol{F}$ (this happens, for instance, when $\boldsymbol{F} \in L^{2}\left(\Gamma_{F} ; \mathbb{R}^{3}\right)$ and then $\widetilde{\boldsymbol{F}} \in L^{2}\left(\Gamma ; \mathbb{R}^{3}\right) \subset H^{-\frac{1}{2}}\left(\Gamma ; \mathbb{R}^{3}\right)$ is any extension to all $\Gamma$ ) and let $V:=\left\{\boldsymbol{v} \in H^{1}\left(\Omega ; \mathbb{R}^{3}\right) ;\left.\boldsymbol{v}\right|_{\Gamma_{0}}=0\right\}$. Let us also assume that $\boldsymbol{u}_{0} \in H^{\frac{1}{2}}\left(\Gamma ; \mathbb{R}^{3}\right)$ and $\operatorname{supp}\left(\boldsymbol{u}_{0}\right) \subseteq \overline{\Gamma_{0}}$. We then define $\Sigma_{a d}$ in the following weak form:

$$
\Sigma_{a d}:=\left\{\boldsymbol{\tau} \in L^{2}\left(\Omega ; \mathbb{M}_{s y m}^{3}\right) ; \int_{\Omega} \boldsymbol{\tau}: \boldsymbol{e}(\boldsymbol{v}) d x-\int_{\Omega} \boldsymbol{f} \boldsymbol{v} d x-\langle\widetilde{\boldsymbol{F}}, \boldsymbol{v}\rangle_{\Gamma}=0 \text { for all } \boldsymbol{v} \in V\right\} .
$$

Let us explicitly remark that if $\boldsymbol{\tau} \in \Sigma_{a d}$ then Div $\boldsymbol{\tau}+\boldsymbol{f}=\mathbf{0}$ in $\Omega$ and hence $\boldsymbol{\tau} \in H($ Div; $\Omega)$, $\boldsymbol{\tau} \mathbf{n} \in H^{-\frac{1}{2}}\left(\Gamma ; \mathbb{R}^{3}\right)$ and $(1)$ holds true. Within this functional framework the complementary energy becomes:

$$
\Psi(\boldsymbol{\tau}):=\frac{1}{2} \int_{\Omega} \boldsymbol{A} \boldsymbol{\tau}: \boldsymbol{\tau} d x-\left\langle\boldsymbol{\tau} \mathbf{n}, \boldsymbol{u}_{0}\right\rangle_{\Gamma}
$$

Hence the stress field $\boldsymbol{\sigma} \in \Sigma_{a d}$ solution of the equilibrium problem realizes the minimum on $\Sigma_{a d}$ of $\Psi(\tau)$. If, as usual in convex analysis, see e.g. [13], $\chi_{\Sigma_{a d}}$ denotes the characteristic function of $\Sigma_{a d}$, then in an equivalent way the stress field $\boldsymbol{\sigma} \in \Sigma_{a d}$ realizes the minimum of

$$
J(\boldsymbol{\tau}):=\frac{1}{2} \int_{\Omega} \boldsymbol{A} \boldsymbol{\tau}: \boldsymbol{\tau} d x-\left\langle\boldsymbol{\tau} \mathbf{n}, \boldsymbol{u}_{0}\right\rangle_{\Gamma}+\chi_{\Sigma_{a d}}(\boldsymbol{\tau})
$$


on $L^{2}\left(\Omega ; \mathbb{M}_{\text {sym }}^{3}\right)$. It is easy to verify that such a minimum $\boldsymbol{\sigma}$ exists and is unique. This means that for all $\boldsymbol{\tau} \in \Sigma_{a d}$ one has :

$$
\int_{\Omega} \boldsymbol{A} \boldsymbol{\sigma}:(\boldsymbol{\tau}-\boldsymbol{\sigma}) d x=\left\langle(\boldsymbol{\tau}-\boldsymbol{\sigma}) \mathbf{n}, \boldsymbol{u}_{0}\right\rangle_{\Gamma}
$$

It remains to prove that there exists $\boldsymbol{u} \in H^{1}\left(\Omega ; \mathbb{R}^{3}\right)$ verifying $\boldsymbol{A} \boldsymbol{\sigma}=\boldsymbol{e}(\boldsymbol{u})$ and realizing, on the set of admissible displacements $U_{a d}:=\left\{\boldsymbol{v} \in H^{1}\left(\Omega ; \mathbb{R}^{3}\right) ;\left.\boldsymbol{v}\right|_{\Gamma}=\boldsymbol{u}_{0}\right\}$, the minimum of the potential energy:

$$
\Phi(\boldsymbol{v}):=\frac{1}{2} \int_{\Omega} \boldsymbol{C} \boldsymbol{e}(\boldsymbol{v}): \boldsymbol{e}(\boldsymbol{v}) d x-\int_{\Omega} \boldsymbol{f} \boldsymbol{v} d x-\langle\widetilde{\boldsymbol{F}}, \boldsymbol{v}\rangle_{\Gamma}
$$

where $\boldsymbol{C}:=\boldsymbol{A}^{-1}$ is the elasticity tensor field. This is a consequence of the following variant of the Donati theorem:

Proposition 1 Let $\mathbb{M}:=\left\{\boldsymbol{\eta} \in L^{2}\left(\Omega ; \mathbb{M}_{\text {sym }}^{3}\right) ; \int_{\Omega} \boldsymbol{\eta}: \boldsymbol{e}(\boldsymbol{v}) d x=0\right.$ for all $\left.\boldsymbol{v} \in V\right\}$ and let $\mathbb{M}^{\perp}:=\{\boldsymbol{e} \in$ $L^{2}\left(\Omega ; \mathbb{M}_{\text {sym }}^{3}\right) ; \int_{\Omega} e: \boldsymbol{\eta} d x=0 \forall \boldsymbol{\eta}$ in $\left.\mathbb{M}\right\}$. Then:

$$
\mathbb{M}^{\perp}=\left\{\boldsymbol{e} \in L^{2}\left(\Omega ; \mathbb{M}_{\text {sym }}^{3}\right) ; \exists \boldsymbol{v} \in V \text { such that } \boldsymbol{e}=\boldsymbol{e}(\boldsymbol{v})\right\}
$$

Proof Let be $\mathbb{X}:=\left\{\boldsymbol{e} \in L^{2}\left(\Omega ; \mathbb{M}_{\text {sym }}^{3}\right) ; \exists \boldsymbol{v} \in V\right.$ such that $\left.\boldsymbol{e}=\boldsymbol{e}(\boldsymbol{v})\right\}$. From the Green formula (1) it follows immediately that $\mathbb{X} \subset \mathbb{M}^{\perp}$ since for all $\tau \in \mathbb{M}$ one has:

$$
\int_{\Omega} \tau: e d x=0
$$

Since $\mathcal{H}^{2}\left(\Gamma_{0}\right)>0$ it follows that $\mathbb{X}$ is a closed subspace of $L^{2}\left(\Omega ; \mathbb{M}_{\text {sym }}^{3}\right)$. Indeed if $\boldsymbol{e}_{n}=\boldsymbol{e}\left(\boldsymbol{v}_{n}\right)$ is a converging sequence in $L^{2}\left(\Omega ; \mathbb{M}_{\text {sym }}^{3}\right)$ then, thanks to the Poincaré and the Korn inequalities $\boldsymbol{v}_{n}$ is a converging sequence of $V$ whose limit is $\boldsymbol{v} \in V$. Hence $\boldsymbol{e}_{n}=\boldsymbol{e}\left(\boldsymbol{v}_{n}\right)$ converges to $\boldsymbol{e}:=\boldsymbol{e}(\boldsymbol{v}) \in \mathbb{X}$. In order to conclude it is sufficient to remark that $\mathbb{X}^{\perp} \subset \mathbb{M}$ since then by virtue of a classical result one has:

$$
\mathbb{M}^{\perp} \subset\left(\mathbb{X}^{\perp}\right)^{\perp}=\mathbb{X}
$$

Thanks to the classical trace theorem, there exists $\widetilde{\boldsymbol{u}}_{0} \in H^{1}\left(\Omega ; \mathbb{R}^{3}\right)$ such that $\left.\widetilde{\boldsymbol{u}}_{0}\right|_{\Gamma}=\boldsymbol{u}_{0}$. Since $\tau, \sigma \in \Sigma_{a d}$ by using the Green formula (1) it follows:

$$
\left\langle(\boldsymbol{\tau}-\boldsymbol{\sigma}) \mathbf{n}, \boldsymbol{u}_{0}\right\rangle_{\Gamma}=\int_{\Omega} \boldsymbol{e}\left(\widetilde{\boldsymbol{u}}_{0}\right):(\boldsymbol{\tau}-\boldsymbol{\sigma}) d x .
$$

Hence (4) implies that for all $\tau \in \Sigma_{a d}$ one has :

$$
\int_{\Omega} \boldsymbol{A} \boldsymbol{\sigma}:(\boldsymbol{\tau}-\boldsymbol{\sigma}) d x=\int_{\Omega} \boldsymbol{e}\left(\widetilde{\boldsymbol{u}}_{0}\right):(\boldsymbol{\tau}-\boldsymbol{\sigma}) d x .
$$

From (2) it follows that $(\boldsymbol{\tau}-\boldsymbol{\sigma}) \in \mathbb{M}$ and so Proposition 1 implies that there exists $\boldsymbol{w} \in V$ such that $\boldsymbol{A} \boldsymbol{\sigma}-\boldsymbol{e}\left(\widetilde{\boldsymbol{u}}_{0}\right)=\boldsymbol{e}(\boldsymbol{w})$. Let us now remark that $\boldsymbol{u}:=\widetilde{\boldsymbol{u}}_{0}+\boldsymbol{w} \in H^{1}\left(\Omega ; \mathbb{R}^{3}\right)$ is an admissible displacement field since $\left.\boldsymbol{u}\right|_{\Gamma}=\boldsymbol{u}_{0}$. Also $\boldsymbol{\sigma}=\boldsymbol{A}^{-1} \boldsymbol{e}(\boldsymbol{u})=\boldsymbol{C} \boldsymbol{e}(\boldsymbol{u}) \in \Sigma_{a d}$ means that:

$$
\int_{\Omega} \boldsymbol{C e}(\boldsymbol{u}): \boldsymbol{e}(\boldsymbol{v}) d x-\int_{\Omega} \boldsymbol{f} \boldsymbol{v} d x-\langle\widetilde{\boldsymbol{F}}, \boldsymbol{v}\rangle_{\Gamma}=0 \text { for all } \boldsymbol{v} \in V
$$

This is equivalent to say that $\boldsymbol{u} \in H^{1}\left(\Omega ; \mathbb{R}^{3}\right)$ realizes the minimum of the potential energy $\Phi(\boldsymbol{v})$ on the set of admissible displacements $U_{a d}$.

Remark 1 Obviously one can consider other type of boundary conditions (e.g. a combination of some components of the stress vector and of the displacement). 


\section{Problem statement}

Let us consider a three-dimensional Euclidian space identified by $\mathbb{R}^{3}$ and such that the three vectors $\mathbf{e}_{i}$ form an orthonormal basis. Let $\Omega^{-}$and $\Omega^{+}$be two disjoint domains with Lipschitz continuous boundaries $\partial \Omega^{+}$and $\partial \Omega^{-}$, whose outer normal is denoted by $\mathbf{n}$. Let $S=\left\{\partial \Omega^{+} \cap \partial \Omega^{-}\right\}^{\circ} \neq \emptyset$ be the interior of the common part of the boundaries which is assumed to be projectable onto the plane $\left\{x_{3}=0\right\}$ and such that $\mathcal{H}^{2}(S)>0$. Let $\Gamma_{0}^{ \pm} \subset \partial \Omega^{ \pm} \backslash \bar{S}$ be relatively open and such that $\overline{\Gamma_{0}^{ \pm}} \cap \bar{S}=\emptyset$ and $\mathcal{H}^{2}\left(\Gamma_{0}^{ \pm}\right)>0$; let also $\Gamma_{F}^{ \pm} \subset \partial \Omega^{ \pm} \backslash \bar{S}$ be relatively open such that $\Gamma_{0}^{ \pm} \bigcap \Gamma_{F}^{ \pm}=\emptyset$ and $S \bigcap \Gamma_{F}^{ \pm}=\emptyset$. We assume at last that $\partial \Omega^{ \pm}=\overline{\Gamma_{F}^{ \pm}} \cup \overline{\Gamma_{0}^{ \pm}} \cup \bar{S}$.

We consider a multimaterial obtained by the insertion between the materials occupying $\Omega^{-}$and $\Omega^{+}$of a third material. For this, $\Omega^{+}$(resp. $\Omega^{-}$) is moved in the $\boldsymbol{e}_{3}$ (resp. $-\boldsymbol{e}_{3}$ ) direction of an amount equal to the half-thickness $\frac{\varepsilon h}{2}$ of the third material where $\varepsilon$ is a small dimensionless parameter and $h$ is a global characteristic length (for example the diameter of $\overline{\Omega^{-}} \cup \overline{\Omega^{+}}$). Then we set $\Omega_{\varepsilon}^{ \pm}:=$ $\left\{\boldsymbol{x} \pm \frac{\varepsilon h}{2} \boldsymbol{e}_{3}, \boldsymbol{x} \in \Omega^{ \pm}\right\}, S_{\varepsilon}^{ \pm}:=\left\{\boldsymbol{x} \pm \frac{\varepsilon h}{2} \boldsymbol{e}_{3}, \boldsymbol{x} \in S\right\}, \Gamma_{0, \varepsilon}^{ \pm}:=\left\{\boldsymbol{x} \pm \frac{\varepsilon h}{2} \boldsymbol{e}_{3}, \boldsymbol{x} \in \Gamma_{0}^{ \pm}\right\}, \Gamma_{F, \varepsilon}^{ \pm}:=\left\{\boldsymbol{x} \pm \frac{\varepsilon h}{2} \boldsymbol{e}_{3}, \boldsymbol{x} \in \Gamma_{F}^{ \pm}\right\}$ and $B_{\varepsilon}:=\left\{\boldsymbol{x}+\varepsilon z \boldsymbol{e}_{3}, \frac{-h}{2}<z<\frac{h}{2}, \boldsymbol{x} \in S\right\}$. At last $\Omega_{\varepsilon}=\Omega_{\varepsilon}^{+} \cup \Omega_{\varepsilon}^{-} \cup B_{\varepsilon} \cup S_{\varepsilon}^{+} \cup S_{\varepsilon}^{-}$will denote the physical reference configuration of the assembly (see figure 1 left).

We suppose that the materials are linearly hyperelastic. With the notations of the previous section we suppose that a displacement $\boldsymbol{u}_{0, \varepsilon} \in H^{\frac{1}{2}}\left(\partial \Omega_{\varepsilon} ; \mathbb{R}^{3}\right)$ is given with $\operatorname{supp}\left(\boldsymbol{u}_{0, \varepsilon}\right) \subseteq\left(\overline{\Gamma_{0, \varepsilon}^{+}} \cup \overline{\Gamma_{0, \varepsilon}^{-}}\right)$and that the structure is submitted on $\Gamma_{F, \varepsilon}:=\Gamma_{F, \varepsilon}^{+} \cup \Gamma_{F, \varepsilon}^{-}$to surface loads $\boldsymbol{F}_{\varepsilon}$ such that there exists $\widetilde{\boldsymbol{F}}_{\varepsilon} \in H^{-\frac{1}{2}}\left(\partial \Omega_{\varepsilon} ; \mathbb{R}^{3}\right)$ with $\left.\widetilde{\boldsymbol{F}}\right|_{\Gamma_{F, \varepsilon}}=\boldsymbol{F}_{\varepsilon}$. Moreover since the external boundary $\Pi_{\varepsilon}:=\left\{\boldsymbol{x}+\varepsilon z \boldsymbol{e}_{3},-\frac{h}{2}<\right.$ $\left.z<\frac{h}{2}, \boldsymbol{x} \in \partial S\right\}$ of $B_{\varepsilon}$ is traction-free $\operatorname{supp}\left(\widetilde{\boldsymbol{F}}_{\varepsilon}\right) \cap\left(\overline{\Pi_{\varepsilon}}\right)=\emptyset$. The structure is also submitted in $\Omega_{\varepsilon}^{+} \cup \Omega_{\varepsilon}^{-}$ to applied body forces $\boldsymbol{f}_{\varepsilon}^{ \pm}$.
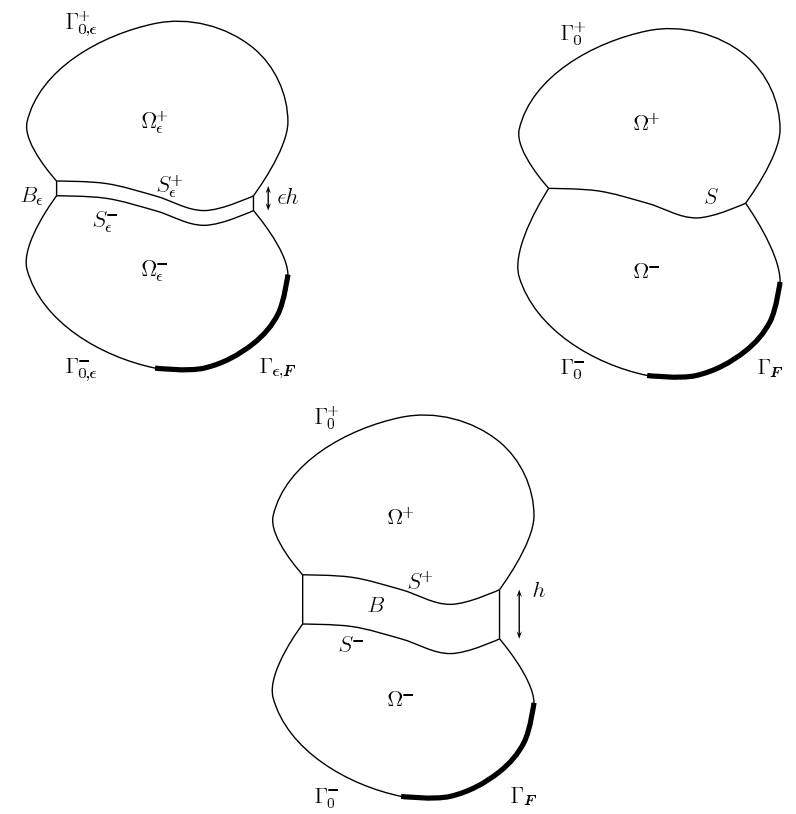

Fig. 1 Left: the physical configuration $\left(\Omega_{\epsilon}\right)$, Right: the limit configuration $(\Omega)$, Below: the rescaled configuration 
In this situation the complementary energy density, $\psi^{\varepsilon}\left(\boldsymbol{x}^{\varepsilon}, \boldsymbol{\tau}^{\varepsilon}\right)$, associated with a stress tensor field $\tau^{\varepsilon}: \Omega_{\varepsilon} \mapsto \mathbb{M}_{\text {sym }}^{3}$, is defined by

$$
\psi^{\varepsilon}\left(\boldsymbol{x}^{\varepsilon}, \boldsymbol{\tau}^{\varepsilon}\right)=\left\{\begin{array}{lc}
\frac{1}{2} \boldsymbol{A}^{+} \boldsymbol{\tau}^{\varepsilon}: \boldsymbol{\tau}^{\varepsilon} & \text { in } \Omega_{\varepsilon}^{+} \\
\frac{1}{2} \boldsymbol{A}^{-} \boldsymbol{\tau}^{\varepsilon}: \boldsymbol{\tau}^{\varepsilon} & \text { in } \Omega_{\varepsilon}^{-} \\
\varepsilon \frac{1}{2} \boldsymbol{A}^{s} \boldsymbol{\tau}^{\varepsilon}: \boldsymbol{\tau}^{\varepsilon} & \text { in } B_{\varepsilon}
\end{array}\right.
$$

and $\boldsymbol{A}^{+}\left(\boldsymbol{x}^{\varepsilon}\right), \boldsymbol{A}^{-}\left(\boldsymbol{x}^{\varepsilon}\right), \boldsymbol{A}^{s}\left(\boldsymbol{x}^{\varepsilon}\right)$ are the hyperelastic fourth-order tensors satisfying the usual symmetry, boundedness and positivity assumptions. Let us explicitly point out that the "adimensional" coefficient $\varepsilon$ in the complementary energy density of the thin layer $B_{\varepsilon}$ expresses the "welding" character of our model.

Using the notations analogous with those of the previous section, the elastic energy associated with a statically admissible stress field $\boldsymbol{\tau}^{\varepsilon}$ is given by the functional

$$
\Psi_{\varepsilon}\left(\boldsymbol{\tau}^{\varepsilon}\right):=\int_{\Omega_{\varepsilon}} \psi^{\varepsilon}\left(\boldsymbol{x}^{\varepsilon}, \boldsymbol{\tau}^{\varepsilon}\right) d x
$$

and the space of the statically admissible stresses is, in the weak form:

$$
\Sigma_{a d}^{\varepsilon}=\left\{\boldsymbol{\tau}^{\varepsilon} \in L^{2}\left(\Omega_{\varepsilon} ; \mathbb{M}_{s y m}^{3}\right) ; \int_{\Omega_{\varepsilon}} \boldsymbol{\tau}^{\varepsilon}: \boldsymbol{e}(\boldsymbol{v}) d x-\int_{\Omega_{\varepsilon}} \boldsymbol{f}_{\varepsilon} \boldsymbol{v} d x-\left\langle\widetilde{\boldsymbol{F}}_{\varepsilon}, \boldsymbol{v}\right\rangle_{\partial \Omega_{\varepsilon}}=0 \text { for all } \boldsymbol{v} \in V^{\varepsilon}\right\}
$$

with $V^{\varepsilon}:=\left\{\boldsymbol{v} \in H^{1}\left(\Omega_{\varepsilon} ; \mathbb{R}^{3}\right)\right.$ with $\left.\left.\boldsymbol{v}\right|_{\Gamma_{0, \varepsilon}}=0\right\}$. Let us explicitly remark that when $\boldsymbol{\tau}^{\varepsilon} \in \Sigma_{a d}^{\varepsilon}$ then Div $\boldsymbol{\tau}^{\varepsilon} \in L^{2}\left(\Omega_{\varepsilon} ; \mathbb{R}^{3}\right)$ and so $\boldsymbol{\tau}^{\varepsilon} \mathbf{n} \in H^{-\frac{1}{2}}\left(\partial \Omega_{\varepsilon} ; \mathbb{R}^{3}\right)$ and the analogous of (1) holds true in $\Omega_{\varepsilon}$.

Hence we can define the complementary energy for all $\tau^{\varepsilon} \in L^{2}\left(\Omega_{\varepsilon} ; \mathbb{M}_{\text {sym }}^{3}\right)$ as:

$$
J_{\varepsilon}\left(\boldsymbol{\tau}^{\varepsilon}\right):=\Psi_{\varepsilon}\left(\boldsymbol{\tau}^{\varepsilon}\right)-\left\langle\boldsymbol{\tau}^{\varepsilon} \mathbf{n}, \boldsymbol{u}_{0, \varepsilon}\right\rangle_{\partial \Omega_{\varepsilon}}+\chi_{\Sigma_{a d}^{\varepsilon}}\left(\boldsymbol{\tau}^{\varepsilon}\right)
$$

where $\langle., \cdot\rangle_{\partial \Omega_{\varepsilon}}$ denotes the duality between $H^{-\frac{1}{2}}\left(\partial \Omega_{\varepsilon} ; \mathbb{R}^{3}\right)$ and $H^{\frac{1}{2}}\left(\partial \Omega_{\varepsilon} ; \mathbb{R}^{3}\right)$. It is easy to verify that there exists a unique $\boldsymbol{\sigma}^{\varepsilon} \in L^{2}\left(\Omega_{\varepsilon} ; \mathbb{M}_{\text {sym }}^{3}\right)$ realizing the minimum of $J_{\varepsilon}$ on $L^{2}\left(\Omega_{\varepsilon} ; \mathbb{M}_{\text {sym }}^{3}\right)$.

As mentioned in the introduction, our aim is to study the behavior of $\boldsymbol{\sigma}^{\varepsilon}:=\operatorname{argmin} J_{\varepsilon}\left(\boldsymbol{\tau}^{\varepsilon}\right)$ when $\varepsilon$ tends to zero and to identify the variational problem whose solution is a suitable limit of $\boldsymbol{\sigma}^{\varepsilon}$. Our strategy consists in computing a suitable variational limit of the sequence $\left(J_{\varepsilon}\right)_{\varepsilon>0}$. In order to make apparent the dependance on $\varepsilon$ of the problem, we define, following the approach of Ciarlet and Destuynder [9], an equivalent problem in the fixed domain $\Omega$ (see figure 1 right). For this purpose, we set $\pi^{\varepsilon}: \boldsymbol{x}=\left(x_{1}, x_{2}, x_{3}\right)=\left(\widehat{x}, x_{3}\right) \in \Omega \rightarrow \boldsymbol{x}^{\varepsilon}=\left(x_{1}^{\varepsilon}, x_{2}^{\varepsilon}, x_{3}^{\varepsilon}\right) \in \Omega_{\varepsilon}$ defined by

$$
\left\{\begin{array}{l}
\pi^{\varepsilon}\left(x_{1}, x_{2}, x_{3}\right)=\left(x_{1}, x_{2}, x_{3}-\frac{h}{2}(1-\varepsilon)\right) \in \Omega_{\varepsilon}^{+}, \quad \text { for } \boldsymbol{x} \in \Omega_{t r}^{+} \\
\pi^{\varepsilon}\left(x_{1}, x_{2}, x_{3}\right)=\left(x_{1}, x_{2}, \varepsilon x_{3}\right) \in B_{\varepsilon}, \quad \text { for } \boldsymbol{x} \in B \\
\pi^{\varepsilon}\left(x_{1}, x_{2}, x_{3}\right)=\left(x_{1}, x_{2}, x_{3}+\frac{h}{2}(1-\varepsilon)\right) \in \Omega_{\varepsilon}^{-}, \quad \text { for } \boldsymbol{x} \in \Omega_{t r}^{-}
\end{array}\right.
$$

with $\Omega_{t r}^{ \pm}=\left\{\boldsymbol{x} \pm \frac{h}{2} \boldsymbol{e}_{3}, \boldsymbol{x} \in \Omega^{ \pm}\right\}, B=\left\{\boldsymbol{x}+z \boldsymbol{e}_{3},-\frac{h}{2}<z<\frac{h}{2}, \boldsymbol{x} \in S\right\}, S^{ \pm}=\left\{\boldsymbol{x} \pm \frac{h}{2} \boldsymbol{e}_{3}, \boldsymbol{x} \in S\right\}$, $\Pi=\left\{\boldsymbol{x}+z \boldsymbol{e}_{3},-\frac{h}{2}<z<\frac{h}{2}, \boldsymbol{x} \in \partial S\right\}$ Let us explicitly remark that since $S^{+}$and $S^{-}$are obtained by a fixed translation in the direction $\boldsymbol{e}_{3}$ from $S$, the Sobolev spaces defined on $S^{+}, S^{-}$and $S$ are isomorphic. In order to simplify the notations, we identify $\Omega_{t r}^{+}$with $\Omega^{+}$and $\Omega_{t r}^{-}$with $\Omega^{-}$and denote by $\Gamma_{0}$, resp. $\Gamma_{F}$, the transformed of $\Gamma_{0, \varepsilon}$, resp. $\Gamma_{F, \varepsilon}$. At last, we set $\Omega=\Omega^{+} \cup \Omega^{-} \cup B \cup S^{+} \cup S^{-}$and we identify $\boldsymbol{\tau}$ with a triple $\left(\boldsymbol{\tau}^{+}, \boldsymbol{\tau}^{-}, \boldsymbol{\tau}^{s}\right) \in L^{2}\left(\Omega^{+} ; \mathbb{M}_{\text {sym }}^{3}\right) \times L^{2}\left(\Omega^{-} ; \mathbb{M}_{\text {sym }}^{3}\right) \times L^{2}\left(B ; \mathbb{M}_{\text {sym }}^{3}\right)$.

The external loads, the elastic properties of the bodies and the stress tensor field in $\Omega^{ \pm}$are defined without rescaling by:

$$
\boldsymbol{\tau}^{ \pm}(\varepsilon, \boldsymbol{x}):=\boldsymbol{\tau}^{\varepsilon, \pm}\left(\boldsymbol{x}^{\varepsilon}\right)=\boldsymbol{\tau}^{\varepsilon, \pm} \circ \pi^{\varepsilon}(\boldsymbol{x}) ; \ldots
$$


and the stress tensor field in $B$ is defined with rescaling:

$$
\boldsymbol{\tau}^{s}(\varepsilon, \boldsymbol{x}):=\boldsymbol{\tau}^{\varepsilon, s}\left(\boldsymbol{x}^{\varepsilon}\right)=\varepsilon \boldsymbol{\tau}^{\varepsilon, s} \circ \pi^{\varepsilon}(\boldsymbol{x})
$$

Let us explicitly remark that thanks to the previous assumptions and with natural notations

$$
\left\langle\boldsymbol{\tau}^{\varepsilon} \mathbf{n}, \boldsymbol{u}_{0, \varepsilon}\right\rangle_{\partial \Omega_{\varepsilon}}=\left\langle\boldsymbol{\tau} \mathbf{n}, \boldsymbol{u}_{0}\right\rangle_{\partial \Omega}, \quad\left\langle\widetilde{\boldsymbol{F}}_{\varepsilon}, \boldsymbol{v}\right\rangle_{\partial \Omega_{\varepsilon}}=\langle\widetilde{\boldsymbol{F}}, \boldsymbol{v}\rangle_{\partial \Omega} .
$$

Let us also recall the usual relations for any differentiable function $\phi^{\varepsilon}$ defined in $B_{\varepsilon}$ :

$$
\begin{gathered}
\frac{\partial}{\partial x_{\alpha}^{\varepsilon}}\left(\phi^{\varepsilon}\left(\boldsymbol{x}^{\varepsilon}\right)\right)=\frac{\partial}{\partial x_{\alpha}}(\phi(\boldsymbol{x})), \\
\frac{\partial}{\partial x_{3}^{\varepsilon}}\left(\phi^{\varepsilon}\left(\boldsymbol{x}^{\varepsilon}\right)\right)=\frac{1}{\varepsilon} \frac{\partial}{\partial x_{3}}(\phi(\boldsymbol{x})),
\end{gathered}
$$

and

$$
\int_{B_{\varepsilon}} \phi^{\varepsilon}\left(\boldsymbol{x}^{\varepsilon}\right) d \boldsymbol{x}^{\varepsilon}=\varepsilon \int_{B} \phi^{\varepsilon} \circ \pi^{\varepsilon}(\boldsymbol{x}) d \boldsymbol{x} \equiv \varepsilon \int_{B} \phi(\boldsymbol{x}) d \boldsymbol{x} .
$$

After this change of coordinates and the rescalings (10),(11), (12), the functional $\Psi^{\varepsilon}$ becomes

$$
\begin{aligned}
& \Psi(\boldsymbol{\tau})=\Psi\left(\boldsymbol{\tau}^{+}, \boldsymbol{\tau}^{-}, \boldsymbol{\tau}^{s}\right)= \\
& \quad \frac{1}{2} \int_{\Omega^{+}} \boldsymbol{A}^{+} \boldsymbol{\tau}^{+}: \boldsymbol{\tau}^{+} d x+\frac{1}{2} \int_{\Omega^{-}} \boldsymbol{A}^{-} \boldsymbol{\tau}^{-}: \boldsymbol{\tau}^{-} d x+\frac{1}{2} \int_{B} \boldsymbol{A}^{s} \boldsymbol{\tau}^{s}: \boldsymbol{\tau}^{s} d x
\end{aligned}
$$

and the weak form of the space of the statically admissible stresses is :

$$
\begin{aligned}
\Sigma_{a d}(\varepsilon): & =\left\{\boldsymbol{\tau}=\left(\boldsymbol{\tau}^{+}, \boldsymbol{\tau}^{-}, \boldsymbol{\tau}^{s}\right) \in L^{2}\left(\Omega^{+} ; \mathbb{M}_{\text {sym }}^{3}\right) \times L^{2}\left(\Omega^{-} ; \mathbb{M}_{s y m}^{3}\right) \times L^{2}\left(B ; \mathbb{M}_{s y m}^{3}\right) ;\right. \\
& \text { for all } \boldsymbol{v}=\left(\boldsymbol{v}^{+}, \boldsymbol{v}^{-}, \boldsymbol{v}^{s}\right) \in V: \\
& \int_{\Omega^{+}} \boldsymbol{\tau}^{+}: \boldsymbol{e}\left(\boldsymbol{v}^{+}\right) d x+\int_{\Omega^{-}} \boldsymbol{\tau}^{-}: \boldsymbol{e}\left(\boldsymbol{v}^{-}\right) d x+\int_{B}\left\{\tau_{\alpha \beta}^{s} e_{\alpha \beta}\left(\boldsymbol{v}^{s}\right)+\tau_{\alpha 3}^{s} v_{3, \alpha}^{s}\right\} d x \\
& \left.+\frac{1}{\varepsilon} \int_{B}\left\{\tau_{\alpha 3}^{s} v_{\alpha, 3}^{s}+\tau_{33}^{s} v_{3,3}^{s}\right\} d x-\int_{\Omega} \boldsymbol{f} \boldsymbol{v} d x-\langle\widetilde{\boldsymbol{F}}, \boldsymbol{v}\rangle_{\partial \Omega}=0\right\}
\end{aligned}
$$

where:

$$
\begin{aligned}
& V:=\left\{\boldsymbol{v}=\left(\boldsymbol{v}^{+}, \boldsymbol{v}^{-}, \boldsymbol{v}^{s}\right) \in H^{1}\left(\Omega^{+} ; \mathbb{R}^{3}\right) \times H^{1}\left(\Omega^{-} ; \mathbb{R}^{3}\right) \times H^{1}\left(B ; \mathbb{R}^{3}\right)\right. \\
& \left.\left.\boldsymbol{v}^{+}\right|_{S^{+}}=\left.\boldsymbol{v}^{s}\right|_{S^{+}},\left.\quad \boldsymbol{v}^{-}\right|_{S^{-}}=\left.\boldsymbol{v}^{s}\right|_{S^{-}},\left.\quad \boldsymbol{v}\right|_{\Gamma_{0}}=0\right\}
\end{aligned}
$$

At last the functional $J_{\varepsilon}\left(\boldsymbol{\tau}^{\varepsilon}\right)$ defined from $L^{2}\left(\Omega^{+} ; \mathbb{M}_{\text {sym }}^{3}\right) \times L^{2}\left(\Omega^{-} ; \mathbb{M}_{\text {sym }}^{3}\right) \times L^{2}\left(B ; \mathbb{M}_{\text {sym }}^{3}\right)$ in $\mathbb{R} \cup\{+\infty\}$ becomes

$$
J(\varepsilon ; \boldsymbol{\tau})=J\left(\varepsilon ; \boldsymbol{\tau}^{+}, \boldsymbol{\tau}^{-}, \boldsymbol{\tau}^{s}\right)=\Psi\left(\boldsymbol{\tau}^{+}, \boldsymbol{\tau}^{-}, \boldsymbol{\tau}^{s}\right)-\left\langle\boldsymbol{\tau} \mathbf{n}, \boldsymbol{u}_{0}\right\rangle_{\partial \Omega}+\chi_{\Sigma_{a d}(\varepsilon)}\left(\boldsymbol{\tau}^{+}, \boldsymbol{\tau}^{-}, \boldsymbol{\tau}^{s}\right) .
$$

Let us stress that $J(\varepsilon ; \boldsymbol{\tau})$ is convex, lower semicontinuous and proper. For further use we explicitly remark that $\widetilde{\boldsymbol{F}} \in H^{-\frac{1}{2}}\left(\partial \Omega ; \mathbb{R}^{3}\right)$ fulfills the condition $\operatorname{supp}(\widetilde{\boldsymbol{F}}) \cap(\bar{\Pi})=\emptyset$. At last, in order to avoid inessential technicalities, in the following we assume that $S$ is indeed contained in the plane $x_{3}=0$. 


\section{Mosco-convergence}

4.1 Preliminaries

The so-called Mosco-convergence was introduced by U.Mosco [21] in 1969 as a useful tool to study the asymptotic behavior of convex functionals.

Definition 1 Let $X$ be a reflexive Banach space, $F_{n}, F: X \longrightarrow \mathbb{R} \cup\{+\infty\}$ convex, lower semicontinuous and proper functionals. The sequence $\left(F_{n}\right)_{n \in \mathbb{N}}$ Mosco-converges to $F$ if and only if:

i) for all $u \in X$ and all sequence $\left(u_{n}\right)_{n \in \mathbb{N}}$ weakly converging to $u$ one has:

$$
\liminf _{n \rightarrow+\infty} F_{n}\left(u_{n}\right) \geq F(u)
$$

ii) for all $u \in X$ there exists a sequence $\left(u_{n}\right)_{n \in \mathbb{N}}$ strongly converging to $u$ in $X$, such that

$$
\limsup _{n \rightarrow+\infty} F_{n}\left(u_{n}\right) \leq F(u) \text {. }
$$

An essential property of Mosco-convergence is that the Young-Fenchel transformation is, for the Moscoconvergence, sequentially bicontinuous from the set $\Gamma(X)$ of convex, lower semicontinuous and proper functionals $F: X \rightarrow \mathbb{R} \cup\{+\infty\}$ onto the set $\Gamma\left(X^{*}\right)$ of convex, lower semicontinuous and proper functionals $F: X^{*} \rightarrow \mathbb{R} \cup\{+\infty\}$, [22]. The proof of this result and of several others interesting properties of Mosco-convergence are given in Chapter 3 of Attouch [2]. Some years later, De Giorgi has introduced the fundamental concept of $\Gamma$-convergence for a very general class of functionals, [11]. As stated in Chapter 3 of Attouch [2], Mosco-convergence of a sequence of functionals defined on a space $X$ is equivalent to $\Gamma$-convergence when $X$ is equipped with its strong and weak topology.

4.2 Mosco-limit of $J(\varepsilon ; \tau)$

Let us consider the convex, lower semicontinuous and proper functional $J: L^{2}\left(\Omega^{+} ; \mathbb{M}_{\text {sym }}^{3}\right) \times L^{2}\left(\Omega^{-} ; \mathbb{M}_{\text {sym }}^{3}\right) \times$ $L^{2}\left(B ; \mathbb{M}_{\text {sym }}^{3}\right) \longrightarrow \mathbb{R} \cup\{+\infty\}$ given by

$$
J(\boldsymbol{\tau})=J\left(\boldsymbol{\tau}^{+}, \boldsymbol{\tau}^{-}, \boldsymbol{\tau}^{s}\right):=\Psi\left(\boldsymbol{\tau}^{+}, \boldsymbol{\tau}^{-}, \boldsymbol{\tau}^{s}\right)-\left\langle\boldsymbol{\tau} \mathbf{n}, \boldsymbol{u}_{0}\right\rangle_{\partial \Omega}+\chi_{\Sigma_{a d}}\left(\boldsymbol{\tau}^{+}, \boldsymbol{\tau}^{-}, \boldsymbol{\tau}^{s}\right)
$$

with

$$
\begin{aligned}
\Sigma_{a d}:= & \left\{\boldsymbol{\tau}=\left(\boldsymbol{\tau}^{+}, \boldsymbol{\tau}^{-}, \boldsymbol{\tau}^{s}\right) \in L^{2}\left(\Omega^{+} ; \mathbb{M}_{\text {sym }}^{3}\right) \times L^{2}\left(\Omega^{-} ; \mathbb{M}_{\text {sym }}^{3}\right) \times L^{2}\left(B ; \mathbb{M}_{\text {sym }}^{3}\right) ;\right. \\
& \tau_{i 3}^{s}=0, \text { and for all } \boldsymbol{v}=\left(\boldsymbol{v}^{+}, \boldsymbol{v}^{-}, \boldsymbol{v}^{s}\right) \in V_{0} \\
& \int_{\Omega_{+}} \boldsymbol{\tau}^{+}: \boldsymbol{e}\left(\boldsymbol{v}^{+}\right) d x+\int_{\Omega_{-}} \boldsymbol{\tau}^{-}: \boldsymbol{e}\left(\boldsymbol{v}^{-}\right) d x+\int_{B} \tau_{\alpha \beta}^{s} e_{\alpha \beta}\left(\boldsymbol{v}^{s}\right) d x \\
& \left.-\int_{\Omega} \boldsymbol{f} \boldsymbol{v} d x-\langle\widetilde{\boldsymbol{F}}, \boldsymbol{v}\rangle_{\partial \Omega}=0\right\}
\end{aligned}
$$

and

$$
V_{0}:=\left\{\boldsymbol{v} \in V, v_{i, 3}^{s}=0\right\} .
$$

Let us remark that when $\tau \in \Sigma_{a d}$ then

$$
\begin{aligned}
& \Psi(\boldsymbol{\tau})=\Psi\left(\boldsymbol{\tau}^{+}, \boldsymbol{\tau}^{-}, \boldsymbol{\tau}^{s}\right)= \\
& \quad \frac{1}{2} \int_{\Omega^{+}} \boldsymbol{A}^{+} \boldsymbol{\tau}^{+}: \boldsymbol{\tau}^{+} d x+\frac{1}{2} \int_{\Omega^{-}} \boldsymbol{A}^{-} \boldsymbol{\tau}^{-}: \boldsymbol{\tau}^{-} d x+\frac{1}{2} \int_{B} A_{\alpha \beta \gamma \delta}^{s} \tau_{\gamma \delta}^{s} \tau_{\alpha \beta}^{s} d x
\end{aligned}
$$


Moreover, since $\boldsymbol{v} \in V_{0}$ implies $\left.\boldsymbol{v}^{+}\right|_{S^{+}}=\left.\boldsymbol{v}^{s}\right|_{S^{+}}=\left.\boldsymbol{v}^{s}\right|_{S^{-}}=\left.\boldsymbol{v}^{-}\right|_{S^{-}}$, it follows also that when $\boldsymbol{\tau} \in \Sigma_{a d}$ one has:

$$
\left.\left\{\left.\left(\boldsymbol{\tau}^{+} \boldsymbol{e}_{3}\right)\right|_{S^{+}} \boldsymbol{e}_{\alpha}-\left.\left(\boldsymbol{\tau}^{-} \boldsymbol{e}_{3}\right)\right|_{S^{-}} \boldsymbol{e}_{\alpha}\right)\right\}=\left.\tau_{\alpha 3}^{+}\right|_{S^{+}}-\left.\tau_{\alpha 3}^{-}\right|_{S^{-}}=-\int_{-1 / 2}^{1 / 2} \tau_{\alpha \beta, \beta}^{s} d x_{3}
$$

for $\alpha=1,2$ and

$$
\left.\tau_{33}^{+}\right|_{S^{+}}=\left.\left(\boldsymbol{\tau}^{+} \boldsymbol{e}_{3}\right)\right|_{S^{+}} \boldsymbol{e}_{3}=\left.\left(\boldsymbol{\tau}^{-} \boldsymbol{e}_{3}\right)\right|_{S^{-}} \boldsymbol{e}_{3}=\left.\tau_{33}^{-}\right|_{S^{-}}
$$

where the equalities have to be understood in $H^{-\frac{1}{2}}(S) \approx H^{-\frac{1}{2}}\left(S^{+}\right) \approx H^{-\frac{1}{2}}\left(S^{-}\right)$.

Theorem 1 Let us equip the space $L^{2}\left(\Omega^{+} ; \mathbb{M}_{\text {sym }}^{3}\right) \times L^{2}\left(\Omega^{-} ; \mathbb{M}_{\text {sym }}^{3}\right) \times L^{2}\left(B ; \mathbb{M}_{\text {sym }}^{3}\right)$ with the standard strong and weak product topologies. Under the previous assumptions, the sequence of functionals $(J(\varepsilon))_{\varepsilon>0}$ Mosco-converges to $J$.

The proof consists in establishing Proposition 2 and Proposition 3 below.

Proposition 2 For all $\boldsymbol{\tau}=\left(\boldsymbol{\tau}^{+}, \boldsymbol{\tau}^{-}, \boldsymbol{\tau}^{s}\right) \in L^{2}\left(\Omega^{+} ; \mathbb{M}_{\text {sym }}^{3}\right) \times L^{2}\left(\Omega^{-} ; \mathbb{M}_{\text {sym }}^{3}\right) \times L^{2}\left(B ; \mathbb{M}_{\text {sym }}^{3}\right)$ and all sequence $\left(\boldsymbol{\tau}^{\varepsilon}\right)_{\varepsilon>0}$ weakly converging to $\boldsymbol{\tau}$ in $L^{2}\left(\Omega^{+} ; \mathbb{M}_{\text {sym }}^{3}\right) \times L^{2}\left(\Omega^{-} ; \mathbb{M}_{\text {sym }}^{3}\right) \times L^{2}\left(B ; \mathbb{M}_{\text {sym }}^{3}\right)$ the following inequality holds:

$$
J(\boldsymbol{\tau}) \leq \liminf _{\varepsilon \rightarrow 0} J\left(\varepsilon ; \boldsymbol{\tau}^{\varepsilon}\right)
$$

Proof Clearly one may assume that $\liminf _{\varepsilon \rightarrow 0} J\left(\varepsilon ; \tau^{\varepsilon}\right)<+\infty$. Therefore for a non relabeled subsequence one has $\tau^{\varepsilon} \in \Sigma_{a d}(\varepsilon)$. Since $\Psi$ is convex one has only to prove that $\tau \in \Sigma_{a d}$. Let us remark at first that for all $\boldsymbol{v} \in V_{0}$ :

$$
\begin{aligned}
& \int_{\Omega_{+}} \boldsymbol{\tau}^{\varepsilon+}: \boldsymbol{e}\left(\boldsymbol{v}^{+}\right) d x+\int_{\Omega_{-}} \boldsymbol{\tau}^{\varepsilon-}: \boldsymbol{e}\left(\boldsymbol{v}^{-}\right) d x+\int_{B}\left\{\tau_{\alpha \beta}^{\varepsilon s} e_{\alpha \beta}\left(\boldsymbol{v}^{s}\right)+\tau_{\alpha 3}^{\varepsilon s} v_{3, \alpha}^{s}\right\} d x \\
& -\int_{\Omega} \boldsymbol{f} \boldsymbol{v} d x-\langle\widetilde{\boldsymbol{F}}, \boldsymbol{v}\rangle_{\partial \Omega}=0
\end{aligned}
$$

and passing to the limit:

$$
\begin{aligned}
& \int_{\Omega_{+}} \boldsymbol{\tau}^{+}: \boldsymbol{e}\left(\boldsymbol{v}^{+}\right) d x+\int_{\Omega_{-}} \boldsymbol{\tau}^{-}: \boldsymbol{e}\left(\boldsymbol{v}^{-}\right) d x+\int_{B}\left\{\tau_{\alpha \beta}^{s} e_{\alpha \beta}\left(\boldsymbol{v}^{s}\right)+\tau_{\alpha 3}^{s} v^{s}{ }_{3}, \alpha\right\} d x \\
& -\int_{\Omega} \boldsymbol{f} \boldsymbol{v} d x-\langle\widetilde{\boldsymbol{F}}, \boldsymbol{v}\rangle_{\partial \Omega}=0 .
\end{aligned}
$$

By multiplying equation (14) by $\varepsilon$ and passing to the limit we find for all $\boldsymbol{v} \in V$ :

$$
\int_{B}\left\{\tau_{\alpha 3}^{s} v_{\alpha, 3}^{s}+\tau_{33}^{s} v_{3,3}^{s}\right\} d x=0
$$

In order to conclude we have to prove that $\tau_{i 3}^{s}=0$. From $(22)$ it follows that $\tau_{i 3,3}^{s}=0$ and so $\tau_{i 3}^{s}$ is constant with respect to $x_{3}$. By choosing $\boldsymbol{v} \in V$ such that $\left.v_{i}^{+}\right|_{S^{+}}=\left.v_{i}^{s}\right|_{S^{+}}=0$ and $\left.v_{i}^{-}\right|_{S^{-}}=\left.v_{i}^{s}\right|_{S^{-}}$is arbitrary in $H^{\frac{1}{2}}\left(S^{-}\right)$and $v_{j}=0$ for $i \neq j$ we get $\tau_{i 3}^{s}=0$. Hence $\tau \in \Sigma_{a d}$.

In order to conclude the proof of Theorem 1 for every element $\tau \in \Sigma_{a d}$ we have to construct a so-called recovery sequence $\left(\boldsymbol{\tau}^{\varepsilon}\right)_{\varepsilon>0} \subset \Sigma_{a d}(\varepsilon)$ strongly converging to $\boldsymbol{\tau}$ in $L^{2}\left(\Omega^{+} ; \mathbb{M}_{\text {sym }}^{3}\right) \times L^{2}\left(\Omega^{-} ; \mathbb{M}_{\text {sym }}^{3}\right) \times$ $L^{2}\left(B ; \mathbb{M}_{\text {sym }}^{3}\right)$. This is the object of Proposition 3 . The following preliminary result will be useful in the proof of Proposition 3.

Lemma 1 Let $\boldsymbol{\tau} \in \Sigma_{\text {ad }}$. There exists a sequence $\left(\boldsymbol{\tau}^{\eta}\right)_{\eta>0}$ in $L^{2}\left(\Omega^{+} ; \mathbb{M}_{\text {sym }}^{3}\right) \times L^{2}\left(\Omega^{-} ; \mathbb{M}_{\text {sym }}^{3}\right) \times L^{2}\left(B ; \mathbb{M}_{\text {sym }}^{3}\right)$ strongly converging to $\tau$ and whose elements satisfy $\tau_{i 3}^{\eta s}=0,(18)$, (19) and moreover are such that $\left(\left.\tau_{i 3}^{ \pm}\right|_{S^{ \pm}}\right)_{\eta} \subset C^{\infty}\left(\overline{S^{ \pm}} ; \mathbb{R}^{3}\right)$ and $\left(\boldsymbol{\tau}^{\eta s}\right)_{\eta} \subset C^{\infty}\left(\bar{B} \backslash\left(S^{+} \cup S^{-}\right) ; \mathbb{M}_{\text {sym }}^{3}\right)$. 
Proof Since $\boldsymbol{\tau} \in \Sigma_{a d}$ then the trace theorem implies that $\boldsymbol{\tau}^{ \pm} \mathbf{n}^{ \pm} \in H^{-\frac{1}{2}}\left(\partial \Omega^{ \pm} ; \mathbb{R}^{3}\right)$ where $\mathbf{n}^{ \pm}$is the outer normal to $\partial \Omega^{ \pm}$. Thanks to the assumptions on $\Omega$ and on $\boldsymbol{F}$ the restrictions $\left.\left(\boldsymbol{\tau}^{ \pm} \boldsymbol{e}_{3}\right)\right|_{S^{ \pm}}$, can be extended by zero in a neighborhood $\widetilde{S}^{ \pm}$of $S^{ \pm}$contained in the plane $x_{3}= \pm \frac{h}{2}$ and these extensions belong to $H^{-\frac{1}{2}}\left(\widetilde{S}^{ \pm} ; \mathbb{R}^{3}\right)$. Let also extend $\boldsymbol{\tau}^{s}$ by zero to $\widetilde{B}=\left\{\boldsymbol{x}+\frac{z}{2} \boldsymbol{e}_{3},-h<z<h, \boldsymbol{x} \in \widetilde{S}\right\}$. One can then make a tangential regularization of such extensions with a sequence of mollifiers depending only on $\widehat{x}$. In this way one constructs sequences $\left(\boldsymbol{g}^{ \pm \eta}\right)_{\eta} \subset C^{\infty}\left(\overline{S^{ \pm}} ; \mathbb{R}^{3}\right)$ converging to $\left.\left(\boldsymbol{\tau}^{ \pm} \boldsymbol{e}_{3}\right)\right|_{S^{ \pm}}$in $H^{-\frac{1}{2}}\left(S^{ \pm} ; \mathbb{R}^{3}\right)$ and $\left(\boldsymbol{\tau}^{\eta s}\right)_{\eta} \subset C^{\infty}\left(\widehat{B}^{s} ; \mathbb{M}_{\text {sym }}^{3}\right)$ converging to $\boldsymbol{\tau}^{s}$ in $L^{2}\left(B ; \mathbb{M}_{\text {sym }}^{3}\right)$ where $\widehat{B}^{s}:=\bar{B} \backslash\left(S^{+} \cup S^{-}\right)=\left\{\boldsymbol{x}+z \boldsymbol{e}_{3},-\frac{h}{2}<\right.$ $\left.z<\frac{h}{2}, \boldsymbol{x} \in \bar{S}\right\}$. These sequences satisfy $\tau_{i 3}^{\eta s}=0$ and the conditions (18), (19). In order to conclude the proof of the lemma we have to construct a suitable lifting $\boldsymbol{\tau}^{ \pm \eta}$ of $\boldsymbol{g}^{ \pm \eta}$. This will be done solving some auxiliary elasticity problems with suitable boundary conditions of mixed type. For every $\eta$ let us consider in $\Omega^{ \pm}$a mixed elasticity problem with an elasticity tensor $\mathbf{C}$ (for simplicity with components $\left.C_{i j k l}=\frac{1}{2}\left(\delta_{i k} \delta_{j l}+\delta_{i l} \delta_{j k}\right)\right)$ where the source terms correspond to $\boldsymbol{f}, \boldsymbol{F}$ and to $\boldsymbol{g}^{ \pm \eta}$ and the zero Dirichlet data is imposed on $\Gamma_{0}^{ \pm}$. These problems have a unique solution $\mathbf{u}^{ \pm \eta} \in H^{1}\left(\Omega^{ \pm} ; \mathbb{R}^{3}\right)$. Let $\boldsymbol{\tau}^{ \pm \eta}:=\mathbf{C e}\left(\boldsymbol{u}^{ \pm \eta}\right) \in L^{2}\left(\Omega^{ \pm} ; \mathbb{M}_{\text {sym }}^{3}\right)$. The continuous dependence on the data implies the convergence of $\boldsymbol{\tau}^{ \pm \eta}$ to $\boldsymbol{\tau}^{ \pm}$in $L^{2}\left(\Omega^{ \pm} ; \mathbb{M}_{\text {sym }}^{3}\right)$. For further use let us point out that the weak formulation of these elasticity problems implies that:

$$
\begin{aligned}
& \int_{\Omega_{+}} \boldsymbol{\tau}^{\eta+}: \boldsymbol{e}\left(\boldsymbol{v}^{+}\right) d x+\int_{\Omega_{-}} \boldsymbol{\tau}^{\eta-}: \boldsymbol{e}\left(\boldsymbol{v}^{-}\right) d x-\int_{\Omega} \boldsymbol{f} \boldsymbol{v} d x-\langle\widetilde{\boldsymbol{F}}, \boldsymbol{v}\rangle_{\partial \Omega} \\
& -\int_{S^{+}} \boldsymbol{g}^{+\eta} \boldsymbol{v}^{+} d \widehat{x}+\int_{S^{-}} \boldsymbol{g}^{-\eta} \boldsymbol{v}^{-} d \widehat{x}=0
\end{aligned}
$$

for all $\left(\boldsymbol{v}^{+}, \boldsymbol{v}^{-}\right) \in H^{1}\left(\Omega^{+} ; \mathbb{R}^{3}\right) \times H^{1}\left(\Omega^{-} ; \mathbb{R}^{3}\right)$ such that $\left.\boldsymbol{v}^{ \pm}\right|_{\Gamma_{0}^{ \pm}}=0$.

Proposition 3 For all $\boldsymbol{\tau}=\left(\boldsymbol{\tau}^{+}, \boldsymbol{\tau}^{-}, \boldsymbol{\tau}^{s}\right) \in L^{2}\left(\Omega^{+} ; \mathbb{M}_{\text {sym }}^{3}\right) \times L^{2}\left(\Omega^{-} ; \mathbb{M}_{\text {sym }}^{3}\right) \times L^{2}\left(B ; \mathbb{M}_{\text {sym }}^{3}\right)$ there exists a sequence $\left(\boldsymbol{\tau}^{\varepsilon}\right)_{\varepsilon>0}$ strongly converging to $\boldsymbol{\tau}$ in $L^{2}\left(\Omega^{+} ; \mathbb{M}_{\text {sym }}^{3}\right) \times L^{2}\left(\Omega^{-} ; \mathbb{M}_{\text {sym }}^{3}\right) \times L^{2}\left(B ; \mathbb{M}_{\text {sym }}^{3}\right)$ such that

$$
\lim _{\varepsilon \rightarrow 0} J\left(\varepsilon ; \boldsymbol{\tau}^{\varepsilon}\right)=J(\boldsymbol{\tau})
$$

Proof Let $\boldsymbol{\tau}$ be a fixed element in $L^{2}\left(\Omega^{+} ; \mathbb{M}_{\text {sym }}^{3}\right) \times L^{2}\left(\Omega^{-} ; \mathbb{M}_{\text {sym }}^{3}\right) \times L^{2}\left(B ; \mathbb{M}_{\text {sym }}^{3}\right)$. One can assume that $J(\boldsymbol{\tau})<\infty$ otherwise there is nothing to prove and hence that $\boldsymbol{\tau} \in \Sigma_{a d}$. Let then be $\left(\boldsymbol{\tau}^{\eta}\right)_{\eta>0}$ a sequence in $L^{2}\left(\Omega^{+} ; \mathbb{M}_{\text {sym }}^{3}\right) \times L^{2}\left(\Omega^{-} ; \mathbb{M}_{\text {sym }}^{3}\right) \times L^{2}\left(B ; \mathbb{M}_{\text {sym }}^{3}\right)$ converging to $\tau$ constructed by using Lemma 1 and hence such that:

$$
\left.\tau_{i 3}^{\eta \pm}\right|_{S^{ \pm}}=g_{i}^{\eta \pm}
$$

Let us now define $\boldsymbol{\tau}^{\varepsilon \eta}=\boldsymbol{\tau}^{\eta}+\varepsilon \widehat{\boldsymbol{\tau}}^{\varepsilon \eta}$ as follows:

$$
\begin{aligned}
\widehat{\tau}^{\varepsilon \eta s} \in L^{2}\left(B ; \mathbb{M}_{s y m}^{3}\right) & \text { is given by } \\
\widehat{\tau}_{\alpha \beta}^{\varepsilon \eta s} & =0, \\
\widehat{\tau}_{\alpha 3}^{\varepsilon \eta s} & =g_{\alpha}^{\eta-}-\int_{-1 / 2}^{x_{3}} \tau_{\alpha \beta, \beta}^{\eta s} d t\left\{=g_{\alpha}^{\eta+}+\int_{x_{3}}^{1 / 2} \tau_{\alpha \beta, \beta}^{\eta s} d t\right\}, \\
\widehat{\tau}_{33}^{\varepsilon \eta s} & =-g_{3}^{\eta-}-\varepsilon \int_{-1 / 2}^{x_{3}} \widehat{\tau}_{\alpha 3, \alpha}^{\varepsilon \eta s} d t ;
\end{aligned}
$$

(ii) $\quad \widehat{\boldsymbol{\tau}}^{\varepsilon \eta+} \in L^{2}\left(\Omega^{+} ; \mathbb{M}_{\text {sym }}^{3}\right)$ satisfies for all $\boldsymbol{v}^{+} \in H^{1}\left(\Omega^{+} ; \mathbb{R}^{3}\right)$ such that $\left.\boldsymbol{v}\right|_{\Gamma_{0}^{+}}=0$ :

$$
\int_{\Omega^{+}} \widehat{\boldsymbol{\tau}}^{\varepsilon \eta+}: \boldsymbol{e}\left(\boldsymbol{v}^{+}\right) d x-\int_{S^{+}}\left\{\int_{-1 / 2}^{1 / 2} \widehat{\tau}_{\alpha 3, \alpha}^{\varepsilon \eta s} d x_{3}\right\} v_{3}^{+} d \widehat{x}=0
$$

(iii) $\quad \widehat{\boldsymbol{\tau}}^{\varepsilon \eta-}=0$. 
Let us explicitly remark that $\widehat{\boldsymbol{\tau}}^{\varepsilon \eta+}$ can be obtained solving a suitable mixed elasticity problem. From the previous choices for every $\varepsilon, \eta>0$ it follows that $\boldsymbol{\tau}^{\varepsilon \eta} \in \Sigma_{a d}(\varepsilon)$. Indeed (23), (29) and (26) imply that for every $\boldsymbol{v} \in V$

$$
\begin{aligned}
& \int_{\Omega^{+}} \boldsymbol{\tau}^{\varepsilon \eta+}: \boldsymbol{e}\left(\boldsymbol{v}^{+}\right) d x+\int_{\Omega^{-}} \boldsymbol{\tau}^{\varepsilon \eta-}: \boldsymbol{e}\left(\boldsymbol{v}^{-}\right) d x+\int_{B}\left\{\tau_{\alpha \beta}^{\varepsilon \eta s} e_{\alpha \beta}\left(\boldsymbol{v}^{s}\right)+\tau_{\alpha 3}^{\varepsilon \eta s} v_{3, \alpha}^{s}\right\} d x \\
& +\frac{1}{\varepsilon} \int_{B}\left\{\tau_{\alpha 3}^{\varepsilon \eta s} v_{\alpha, 3}^{s}+\tau_{33}^{\varepsilon \eta s} v_{3,3}^{s}\right\} d x-\int_{\Omega} \boldsymbol{f} \boldsymbol{v} d x-\langle\widetilde{\boldsymbol{F}}, \boldsymbol{v}\rangle_{\partial \Omega} \\
& =\left.\int_{S^{+}}\left(\boldsymbol{g}^{\eta+} \boldsymbol{e}_{3}\right)\right|_{S^{+}} \boldsymbol{v}^{+} d \widehat{x}-\left.\int_{S^{-}}\left(\boldsymbol{g}^{\eta-} \boldsymbol{e}_{3}\right)\right|_{S^{-}} \boldsymbol{v}^{-} d \widehat{x} \\
& +\varepsilon\left\{\int_{S^{+}}\left\{\int_{-1 / 2}^{1 / 2} \widehat{\tau}_{\alpha 3, \alpha}^{\varepsilon \eta s} d x_{3}\right\} v_{3}^{+} d \widehat{x}\right\} \\
& +\int_{B}\left\{\tau_{\alpha \beta}^{\eta s} e_{\alpha \beta}\left(\boldsymbol{v}^{s}\right)+\widehat{\tau}_{\alpha 3}^{\varepsilon \eta s} v_{\alpha, 3}^{s}\right\} d x+\int_{B}\left\{\varepsilon \widehat{\tau}_{\alpha 3}^{\varepsilon \eta s} v_{3, \alpha}^{s}+\widehat{\tau}_{33}^{\varepsilon \eta s} v_{3,3}^{s}\right\} d x \\
& =\int_{B}\left\{\tau_{\alpha \beta}^{\eta s} e_{\alpha \beta}\left(\boldsymbol{v}^{s}\right)+\widehat{\tau}_{\alpha 3}^{\varepsilon \eta n} v_{\alpha, 3}^{s}\right\} d x+\int_{S^{+}} g_{\alpha}^{\eta+} v_{\alpha}^{+} d \widehat{x}-\int_{S^{-}} g_{\alpha}^{\eta-} v_{\alpha}^{-} d \widehat{x} \\
& +\varepsilon\left(\int_{S^{+}}\left\{\int_{-1 / 2}^{1 / 2} \widehat{\tau}_{\alpha 3, \alpha}^{\varepsilon \eta s} d x_{3}\right\} v_{3}^{+} d \widehat{x}\right)+\int_{S^{+}} g_{3}^{\eta+} v_{3}^{+} d \widehat{x}-\int_{S^{-}} g_{3}^{\eta-} v_{3}^{-} d \widehat{x} \\
& +\int_{B}\left\{\varepsilon \widehat{\tau}_{\alpha 3}^{\varepsilon \eta s} v_{3, \alpha}^{s}+\widehat{\tau}_{33}^{\varepsilon \eta s} v_{3,3}^{s}\right\} d x=0,
\end{aligned}
$$

since from (27) it follows that

$$
\int_{B}\left\{\tau_{\alpha \beta}^{\eta s} e_{\alpha \beta}\left(\boldsymbol{v}^{s}\right)+\widehat{\tau}_{\alpha 3}^{\varepsilon \eta s} v_{\alpha, 3}^{s}\right\} d x+\int_{S^{+}} g_{\alpha}^{\eta+} v_{\alpha}^{+} d \widehat{x}-\int_{S^{-}} g_{\alpha}^{\eta-} v_{\alpha}^{+} d \widehat{x}=0
$$

and from (28) it follows that

$$
\begin{aligned}
& +\varepsilon \int_{S^{+}}\left\{\int_{-1 / 2}^{1 / 2} \widehat{\tau}_{\alpha 3, \alpha}^{\varepsilon \eta s} d x_{3}\right\} v_{3}^{+} d \widehat{x}+\int_{S^{+}} g_{3}^{\eta+} v_{3}^{+} d \widehat{x}-\int_{S^{-}} g_{3}^{\eta-} v_{3}^{-} d \widehat{x} \\
& +\int_{B}\left\{\varepsilon \widehat{\tau}_{\alpha 3}^{\varepsilon \eta s} v_{3, \alpha}^{s}+\widehat{\tau}_{33}^{\varepsilon \eta s} v_{3,3}^{s}\right\} d x=0 .
\end{aligned}
$$

Since

$$
\lim _{\varepsilon \rightarrow 0} J\left(\varepsilon ; \boldsymbol{\tau}^{\varepsilon \eta}\right)=\Psi\left(\boldsymbol{\tau}^{\eta}\right)
$$

then

$$
\lim _{\eta \rightarrow 0} \lim _{\varepsilon \rightarrow 0} J\left(\varepsilon ; \boldsymbol{\tau}^{\varepsilon \eta}\right)=\Psi(\boldsymbol{\tau})=J(\boldsymbol{\tau}) .
$$

From the construction of $\boldsymbol{\tau}^{\varepsilon \eta}$ it follows that $\lim _{\eta \rightarrow 0} \lim _{\varepsilon \rightarrow 0} \boldsymbol{\tau}^{\varepsilon \eta}=\boldsymbol{\tau}$ strongly in $L^{2}\left(\Omega^{+} ; \mathbb{M}_{\text {sym }}^{3}\right) \times$ $L^{2}\left(\Omega^{-} ; \mathbb{M}_{\text {sym }}^{3}\right) \times L^{2}\left(B ; \mathbb{M}_{\text {sym }}^{3}\right)$. Hence, thanks to a diagonalization lemma (see e.g. Attouch [2]), there exists a map $\varepsilon \rightarrow \eta(\varepsilon)$ such that $\eta(\varepsilon) \rightarrow 0$ when $\varepsilon \rightarrow 0$ and such that

$$
\lim _{\varepsilon \rightarrow 0} J\left(\varepsilon ; \boldsymbol{\tau}^{\varepsilon \eta(\varepsilon)}\right)=J(\boldsymbol{\tau}) \quad \text { and } \quad \lim _{\varepsilon \rightarrow 0} \boldsymbol{\tau}^{\varepsilon \eta(\varepsilon)}=\boldsymbol{\tau} .
$$




\section{A Donati-type theorem}

Let us remark at first that the functional $J(\boldsymbol{\tau})=J\left(\boldsymbol{\tau}^{+}, \boldsymbol{\tau}^{-}, \boldsymbol{\tau}^{s}\right)$ has a minimum $\boldsymbol{\sigma}=\left(\boldsymbol{\sigma}^{+}, \boldsymbol{\sigma}^{-}, \boldsymbol{\sigma}^{s}\right) \in$ $\Sigma_{a d}$ on $L^{2}\left(\Omega^{+} ; \mathbb{M}_{\text {sym }}^{3}\right) \times L^{2}\left(\Omega^{-} ; \mathbb{M}_{\text {sym }}^{3}\right) \times L^{2}\left(B ; \mathbb{M}_{\text {sym }}^{3}\right)$; this means that

$$
\begin{aligned}
& \int_{\Omega^{+}} \boldsymbol{A}^{+} \boldsymbol{\sigma}^{+}:\left(\boldsymbol{\tau}^{+}-\boldsymbol{\sigma}^{+}\right) d x+\int_{\Omega^{-}} \boldsymbol{A}^{-} \boldsymbol{\sigma}^{-}:\left(\boldsymbol{\tau}^{-}-\boldsymbol{\sigma}^{-}\right) d x \\
& +\int_{B} A_{\alpha \beta \gamma \delta}^{s} \sigma_{\gamma \delta}^{s}:\left(\tau_{\alpha \beta}^{s}-\sigma_{\alpha \beta}^{s}\right) d x=\left\langle(\boldsymbol{\tau}-\boldsymbol{\sigma}) \mathbf{n}, \boldsymbol{u}_{0}\right\rangle_{\partial \Omega} \text { for all } \boldsymbol{\tau}=\left(\boldsymbol{\tau}^{+}, \boldsymbol{\tau}^{-}, \boldsymbol{\tau}^{s}\right) \in \Sigma_{a d} .
\end{aligned}
$$

Thanks to the classical trace theorem there exists $\widetilde{\boldsymbol{u}}_{0}=\left(\widetilde{\boldsymbol{u}}_{0}^{+}, \widetilde{\boldsymbol{u}}_{0}^{-}, \widetilde{\boldsymbol{u}}_{0}^{s}\right) \in H^{1}\left(\Omega ; \mathbb{R}^{3}\right)$ with $\widetilde{\boldsymbol{u}}_{0}^{s}=\mathbf{0}$ such that $\left.\widetilde{\boldsymbol{u}}_{0}\right|_{\partial \Omega}=\boldsymbol{u}_{0}$ and hence from the Green formula (1) it follows:

$$
\left\langle(\boldsymbol{\tau}-\boldsymbol{\sigma}) \mathbf{n}, \boldsymbol{u}_{0}\right\rangle_{\partial \Omega}=\int_{\Omega} \boldsymbol{e}\left(\widetilde{\boldsymbol{u}}_{0}\right):(\boldsymbol{\tau}-\boldsymbol{\sigma}) d x
$$

Let

$$
\begin{gathered}
\mathbb{M}_{0}:=\left\{\boldsymbol{\eta}=\left(\boldsymbol{\eta}^{+}, \boldsymbol{\eta}^{-}, \widehat{\boldsymbol{\eta}}^{s}\right) \in L^{2}\left(\Omega^{+} ; \mathbb{M}_{\text {sym }}^{3}\right) \times L^{2}\left(\Omega^{-} ; \mathbb{M}_{\text {sym }}^{3}\right) \times L^{2}\left(B ; \mathbb{M}_{\text {sym }}^{2}\right)\right. \\
\left.\int_{\Omega_{+}} \boldsymbol{\eta}^{+}: \boldsymbol{e}\left(\boldsymbol{v}^{+}\right) d x+\int_{\Omega_{-}} \boldsymbol{\eta}^{-}: \boldsymbol{e}\left(\boldsymbol{v}^{-}\right) d x+\int_{B} \widehat{\eta}_{\alpha \beta}^{s} e_{\alpha \beta}\left(\boldsymbol{v}^{s}\right) d x=0 \text { for all } \boldsymbol{v} \in V_{0}\right\} .
\end{gathered}
$$

Thanks to (32) one can write (31) for all $\boldsymbol{\eta} \in \mathbb{M}_{0}$ as follows:

$$
\int_{\Omega^{+}} \boldsymbol{A}^{+} \boldsymbol{\sigma}^{+}: \boldsymbol{\eta}^{+} d x+\int_{\Omega^{-}} \boldsymbol{A}^{-} \boldsymbol{\sigma}^{-}: \boldsymbol{\eta}^{-} d x+\int_{B} A_{\alpha \beta \gamma \delta}^{s} \sigma_{\gamma \delta}^{s} \widehat{\eta}_{\alpha \beta}^{s} d x=\int_{\Omega} \boldsymbol{e}\left(\widetilde{\boldsymbol{u}}_{0}\right): \boldsymbol{\eta} d x .
$$

This means that $\left(\boldsymbol{A}^{+} \boldsymbol{\sigma}^{+}-\boldsymbol{e}\left(\widetilde{\boldsymbol{u}}_{0}^{+}\right), \boldsymbol{A}^{-} \boldsymbol{\sigma}^{-}-\boldsymbol{e}\left(\widetilde{\boldsymbol{u}}_{0}^{-}\right), \widehat{\boldsymbol{A}^{s}} \widehat{\boldsymbol{\sigma}}^{s}\right) \in \mathbb{M}_{0}^{\perp}$ where $\left(\widehat{\boldsymbol{A}}^{s}\right)_{\alpha \beta}:=A_{\alpha \beta \gamma \delta}^{s},\left(\widehat{\boldsymbol{\sigma}}^{s}\right)_{\gamma \delta}:=\sigma_{\gamma \delta}^{s}$ and the orthogonality is taken with respect to the scalar product in $L^{2}\left(\Omega^{+} ; \mathbb{M}_{\text {sym }}^{3}\right) \times L^{2}\left(\Omega^{-} ; \mathbb{M}_{\text {sym }}^{3}\right) \times$ $L^{2}\left(B ; \mathbb{M}_{\text {sym }}^{2}\right)$. Hence we need to identify $\mathbb{M}_{0}^{\perp}$. This is a consequence of the following variant of the Donati theorem.

Theorem $2 \boldsymbol{e}=\left(\boldsymbol{e}^{+}, \boldsymbol{e}^{-}, \widehat{\boldsymbol{e}}^{s}\right) \in \mathbb{M}_{0}^{\perp}$ if and only if there exists $\boldsymbol{v}=\left(\boldsymbol{v}^{+}, \boldsymbol{v}^{-}, \boldsymbol{v}^{s}\right) \in V_{0}$ such that $\boldsymbol{e}^{+}=\boldsymbol{e}\left(\boldsymbol{v}^{+}\right), \boldsymbol{e}^{-}=\boldsymbol{e}\left(\boldsymbol{v}^{-}\right)$and $\left(\widehat{e}^{s}\right)_{\alpha \beta}=e_{\alpha \beta}\left(\boldsymbol{v}^{s}\right)$.

Proof Let $\mathbb{X}_{0}$ be the subspace of $L^{2}\left(\Omega^{+} ; \mathbb{M}_{\text {sym }}^{3}\right) \times L^{2}\left(\Omega^{-} ; \mathbb{M}_{\text {sym }}^{3}\right) \times L^{2}\left(B ; \mathbb{M}_{\text {sym }}^{2}\right)$ of $\boldsymbol{e}$ such that there exists $\boldsymbol{v}=\left(\boldsymbol{v}^{+}, \boldsymbol{v}^{-}, \boldsymbol{v}^{s}\right) \in V_{0}$ satisfying $\boldsymbol{e}^{+}=\boldsymbol{e}\left(\boldsymbol{v}^{+}\right), \boldsymbol{e}^{-}=\boldsymbol{e}\left(\boldsymbol{v}^{-}\right)$and $\left(\widehat{e}^{s}\right)_{\alpha \beta}=e_{\alpha \beta}\left(\boldsymbol{v}^{s}\right)$. From this definition it follows immediately that $\mathbb{X}_{0} \subset \mathbb{M}_{0}^{\perp}$. We claim that $\mathbb{X}_{0}$ is a closed subspace. Indeed if $\left(\boldsymbol{e}_{n}\right)=\left(\boldsymbol{e}\left(\boldsymbol{v}_{n}^{+}\right), \boldsymbol{e}\left(\boldsymbol{v}_{n}^{-}\right),\left(e_{\alpha \beta}\left(\boldsymbol{v}_{n}^{s}\right)\right)\right)$ is a sequence in $\mathbb{X}_{0}$ converging to $\mathbf{0}$ then the sequence $\left(\boldsymbol{v}_{n}^{+}\right)$, resp. $\left(\boldsymbol{v}_{n}^{-}\right)$, converges in $H^{1}\left(\Omega^{+} ; \mathbb{R}^{3}\right)$, resp. $H^{1}\left(\Omega^{-} ; \mathbb{R}^{3}\right)$, to $\boldsymbol{R}^{+}$, resp. $\boldsymbol{R}^{-}$with $\boldsymbol{R}^{+}$and $\boldsymbol{R}^{-}$rigid displacements. As far as it concerns the sequence $\left(\boldsymbol{v}_{n}^{s}\right)$, whose elements are independent from $x_{3}$, we deduce only that the sequence converges to a displacement $\boldsymbol{R}^{s}=\left(a_{1}-b_{1} x_{2}, a_{2}-b_{2} x_{1}, a_{3}\left(x_{1}, x_{2}\right)\right)$. However the definition of $V_{0}$ implies that $\boldsymbol{R}^{+}=\boldsymbol{R}^{-}=\boldsymbol{R}^{s}=\mathbf{0}$ and the claim is proved. In order to conclude it is now enough to prove that

$$
\mathbb{X}_{0}^{\perp} \subset \mathbb{M}_{0}
$$

since then $\mathbb{M}_{0}^{\perp} \subset\left(\mathbb{X}_{0}^{\perp}\right)^{\perp}=\mathbb{X}_{0}$. For this let be $\boldsymbol{\tau}=\left(\boldsymbol{\tau}^{+}, \boldsymbol{\tau}^{-}, \widehat{\boldsymbol{\tau}}^{s}\right) \in \mathbb{X}_{0}^{\perp}$, i. e. such that

$$
\int_{\Omega_{+}} \boldsymbol{\tau}^{+}: \boldsymbol{e}\left(\boldsymbol{v}^{+}\right) d x+\int_{\Omega_{-}} \boldsymbol{\tau}^{-}: \boldsymbol{e}\left(\boldsymbol{v}^{-}\right) d x+\int_{B} \widehat{\tau}_{\alpha \beta}^{s} e_{\alpha \beta}\left(\boldsymbol{v}^{s}\right) d x=0
$$

for all $\boldsymbol{v} \in V_{0}$. It is then obvious that $\boldsymbol{\tau} \in \mathbb{M}_{0}$.

Corollary 1 There exists $\boldsymbol{u}=\left(\boldsymbol{u}^{+}, \boldsymbol{u}^{-}, \boldsymbol{u}^{s}\right) \in H^{1}\left(\Omega ; \mathbb{R}^{3}\right)$ such that

$$
\left(\boldsymbol{A}^{+} \boldsymbol{\sigma}^{+}, \boldsymbol{A}^{-} \boldsymbol{\sigma}^{-}, \widehat{\boldsymbol{A}}^{s} \widehat{\boldsymbol{\sigma}}^{s}\right)=\left(\boldsymbol{e}\left(\boldsymbol{u}^{+}\right), \boldsymbol{e}\left(\boldsymbol{u}^{-}\right),\left(e_{\alpha \beta}\left(\boldsymbol{u}^{s}\right)\right)\right) .
$$


Proof Theorem 2 implies that there exists $\boldsymbol{w}=\left(\boldsymbol{w}^{+}, \boldsymbol{w}^{-}, \boldsymbol{w}^{s}\right) \in V_{0}$ such that

$$
\left(\boldsymbol{A}^{+} \boldsymbol{\sigma}^{+}-\boldsymbol{e}\left(\widetilde{\boldsymbol{u}}_{0}^{+}\right), \boldsymbol{A}^{-} \boldsymbol{\sigma}^{-}-\boldsymbol{e}\left(\widetilde{\boldsymbol{u}}_{0}^{-}\right), \widehat{\boldsymbol{A}}^{s} \widehat{\boldsymbol{\sigma}}^{s}\right)=\left(\boldsymbol{e}\left(\boldsymbol{w}^{+}\right), \boldsymbol{e}\left(\boldsymbol{w}^{-}\right),\left(e_{\alpha \beta}\left(\boldsymbol{w}^{s}\right)\right)\right)
$$

hence setting $\boldsymbol{u}=\boldsymbol{w}+\widetilde{\boldsymbol{u}}_{0},(36)$ is satisfied.

Let us denote by $\boldsymbol{C}^{+}$and $\boldsymbol{C}^{-}$the inverses (as linear operators in $\mathbb{M}_{\text {sym }}^{3}$ ) of $\boldsymbol{A}^{+}$and $\boldsymbol{A}^{-}$and by $\widehat{\boldsymbol{C}}^{s}$ the operator inverse in $\mathbb{M}_{\text {sym }}^{2}$ of $\widehat{\boldsymbol{A}}^{s}$. Then (15) implies that $\boldsymbol{u}$ realizes the minimum in $\left(\widetilde{\boldsymbol{u}}_{0}+V_{0}\right) \subset$ $H^{1}\left(\Omega ; \mathbb{R}^{3}\right)$ of the functional:

$$
\begin{aligned}
& \Phi(\boldsymbol{v})=\Phi\left(\boldsymbol{v}^{+}, \boldsymbol{v}^{-}, \boldsymbol{v}^{s}\right)=\frac{1}{2} \int_{\Omega^{+}} \boldsymbol{C}^{+} \boldsymbol{e}\left(\boldsymbol{v}^{+}\right): \boldsymbol{e}\left(\boldsymbol{v}^{+}\right) d x+\frac{1}{2} \int_{\Omega^{-}} \boldsymbol{C}^{-} \boldsymbol{e}\left(\boldsymbol{v}^{-}\right): \boldsymbol{e}\left(\boldsymbol{v}^{-}\right) d x \\
& +\frac{1}{2} \int_{B} \widehat{C}_{\alpha \beta \gamma \delta}^{s} e_{\gamma \delta}\left(\boldsymbol{v}^{s}\right) e_{\alpha \beta}\left(\boldsymbol{v}^{s}\right) d x-\int_{\Omega} \boldsymbol{f} \boldsymbol{v} d x-\langle\boldsymbol{F}, \boldsymbol{v}\rangle_{\Gamma} .
\end{aligned}
$$

When $\widetilde{\boldsymbol{u}}_{0}=\mathbf{0}$ this functional coincides with that introduced in [5].

\section{References}

1. Acerbi E., Buttazzo G., Percivale P. : Thin inclusions in linear elasticity : a variational approach. J. Reine Angew. Math., 386, 99-115, (1988)

2. Attouch H . Variational Convergence for Functions and Operators. Applicable Mathematics Series. Pitman Advanced Publishing Program, (1985)

3. Aze D.:Convergence des variables duales dans des problèmes de transmission à travers des couches minces par des méthodes d'épi-convergence. Ricerche di Matematica, 35, 125-159 (1986)

4. Benvenuto E. , An Introduction to the History of Structural Mechanics, Part II: Vaulted Structures and Elastic Systems, Springer-Verlag, (1991)

5. Bessoud A.-L., Krasucki F., Michaille G., Multi-materials with strong interface: variational modelings. Asymptot. Anal., 61 , 1-19, (2008)

6. Brezis H., Caffarelli L.A., Friedman A.: Reinforcement problems for elliptic equations and variational inequalities. Ann. Mat. Pura Appl., 4, 123, 219-246, (1980)

7. Caillerie D. : The effect of a thin inclusion of high rigidity in an elastic body. Math. Methods Appl. Sci., 2, 251-270, (1980)

8. Ciarlet P.G., Ciarlet P. Jr., Iosifescu O., Sauter S., Jun Zou: Lagrange multipliers in intrinsic elasticity. Math. Models Methods Appl. Sci, 21, 651-666, (2011)

9. Ciarlet P.G., Destuynder P.: A justification of the two-dimensional linear plate model. J. Mécanique, 18, 315-344, (1979)

10. Ciarlet P.G., Le Dret H., Nzengwa R. : Junctions between three-dimensional and two-dimensional linearly elastic structures. J. Math. Pures Appl., 68, 261-295,(1989)

11. De Giorgi E., Franzoni T. : Su un tipo di convergenza variazionale. Atti Accad. Naz. Lincei Rend. Cl. Sci. Fis. Mat. Natur. 58 , 842-850, (1975)

12. Duvaut G., Lions J. L. : Les Inéquations en Mécanique et en Physique. Dunod, (1972)

13. Ekeland I.,Temam R.: Analyse Convexe et Problèmes Variationnels. Dunod \& Gauthier-Villars, Paris (1974).

14. Geymonat G., Krasucki F : A limit problem of a soft thin joint. Partial Differential Equations and Applications, (Marcellini P. , Talenti G. , and Vesentini E. , Editors), 165-173. Marcel Dekker, New York, (1996)

15. Geymonat G., Krasucki F : Some remarks on the compatibility conditions in elasticity. Accad. Naz. Sci. XL. 123, 175-182, (2005)

16. Geymonat G., Krasucki F., Lenci S. : Mathematical analysis of a bounded joint with a soft thin adhesive. Math. Mech. Solids, 4, 201-225, (1999)

17. Geymonat G., Suquet P. : Functional Spaces for Norton-Hoff Materials. Math. Meth. in the Appl. Sci., 8, 206-222, (1986) 
18. M. Gurtin :The Linear Theory of Elasticity. Handbuch der Physik, Vol. VI a/2, (S. Flügge and C. Truesdell, Editors), 1-295, Springer-Verlag, (1972)

19. Le Dret H. : Problemes Variationnels dans les Multi-Domaines. Masson, Paris, (1991)

20. Licht C., Michaille G. : A modelling of elastic adhesive bonding joints. Advances in Mathematical Sciences and Applications, 7, 711-740, (1997)

21. Mosco U. : Convergence of convex sets and of solutions of variational inequalities. Adv. in Math. 3, 510-585,(1969)

22. Mosco U. : On the continuity of Young-Fenchel transformation. Journ. Math. Anal. Appl. 35, 518-535, (1971)

23. Pham Huy, H., Sanchez-Palencia, E. : Phénomène de transmission à travers des couches minces de conductivité élevée. J. Math. Anal. Appl., 47, 284-309 (1974)

24. Suquet P. : Discontinuities and plasticity. Non-Smooth Mechanics and Applications, (J. J. Moreau and P. D. Panagiotopoulos, editors) CISM Courses and Lectures, 280-340. Springer-Verlag, Wien, (1988) 